\title{
A model for low-frequency bursts in subthalamic nucleus neurons
}

\author{
Shigeru Kubota* \\ Graduate School of Science and Engineering, Yamagata University, \\ 4-3-16 Jonan, Yonezawa, Yamagata, 992-8510, Japan \\ Jonathan E. Rubin \\ Department of Mathematics, University of Pittsburgh, \\ 301 Thackeray Hall, Pittsburgh, PA 15260, USA \\ *Corresponding author.E-mail: kubota@yz.yamagata-u.ac.jp \\ Tel: +81-238-26-3766, Fax: +81-238-26-3299
}

\begin{abstract}
Burst firing in subthalamic nucleus (STN) has been suggested to be highly associated with the motor symptoms in Parkinson's disease, which result from a loss of dopamine. Although it is clinically very important to clarify the mechanism underlying the bursting dynamics, complex interactions between STN and other brain areas make it difficult to understand. In anesthetized rats, STN neurons exhibit low-frequency ( $\sqcup 1 \mathrm{~Hz}$ ) bursts, which are synchronous with cortical slow oscillations and are significantly strengthened by dopamine depletion. To reproduce these low-frequency bursts, we examine a conductance-based model of an STN neuron that includes NMDA-type glutamatergic inputs reflecting cortical oscillations. In addition, the neuron model contains GABAergic inhibitory inputs, which are assumed to result from the activities of globus pallidus (GP). We show that the STN neuron model can reproduce low-frequency bursts synchronized with cortical activity, in the presence of GABAergic inhibition. In addition, we demonstrate that increased GABA activity leads to enhanced burstiness whereas increased NMDA conductances mainly augment STN firing rate. The induction of burst firing additionally decreases the coherence between STN and cortical activities. These results may give insights into how the complicated interactions between the STN, cortex, and GP can modulate the dynamics of bursting oscillations in the basal ganglia.
\end{abstract}

Keywords: subthalamic nucleus, cortex, globus pallidus, Parkinson's disease, burst oscillations

\section{Introduction}

The subthalamic nucleus (STN) is a key component of the basal ganglia, a group of subcortical brain nuclei involved in motor control. ${ }^{1}$ Bursting oscillations in the STN are suggested to be highly associated with motor disabilities in Parkinson's disease., ${ }^{2,3}$ Although it is clinically important to clarify the mechanisms underlying bursting dynamics, complex interactions between STN and other brain regions complicate efforts to do so.

Experiments using anesthetized rats have shown that STN neurons exhibit low-frequency bursts that are synchronized with cortical slow-wave activity $(\sqcup 1$ 
Hz). ${ }^{4,5}$ Cortical ablation abolished these burst oscillations and produced nearly regular firing in STN. This observation implies that the cortical inputs to STN, which are transmitted through the hyperdirect corticosubthalamic pathway, play a critical role in maintaining the oscillatory component of STN activity. Furthermore, in the case of pharmacological dopamine depletion, a condition that mimics a parkinsonian state, the firing rate of STN neurons was significantly increased to more than twice its original value. ${ }^{5}$ The loss of dopamine also evoked low-frequency oscillations in the GABAergic interneurons in globus pallidus (GP), which are reciprocally connected with STN. ${ }^{5}$ These data may suggest that the low-frequency bursting in STN is considerably modulated by GABA inhibition originating from GP interneurons, in addition to oscillatory inputs from the cortex.

In this study, we simulate a model STN neuron to study how STN bursts depend on cortical inputs, mediated by NMDA currents, and GABAergic inhibition. In the model, synaptic NMDA currents are activated by slow cortical oscillations consisting of active phases of high-frequency activity alternating with inactive quiescent phases, as observed in experiments. ${ }^{6-8}$ We examine the changes in burst properties that arise with changes in the magnitudes of both the NMDA and GABA conductances. We show that enhanced GABAergic inhibition leads to stronger burstiness while enhanced NMDA activation mainly acts to increase STN firing rate. In addition, the occurrence of strong bursts is correlated with a decrease in coherence between the STN and cortical activities. These results may provide insights on how the complex interaction between STN and other associated brain areas modulates dynamical properties of bursting oscillations in the basal ganglia.

\section{Methods}

\subsection{STN neuron model}

We constructed a conductance-based model STN neuron including synaptic NMDA and GABA currents. The NMDA conductance is stochastically activated by the arrival of cortical inputs, while the GABA conductance has a temporally constant magnitude. The membrane potential $V$ of the model neuron is described as

$$
\begin{aligned}
C \frac{d V}{d t}= & -I_{\text {leak }}-I_{N a}-I_{K}-I_{T}-I_{C a}-I_{A H P} \\
& -I_{D I C}-I_{N M D A}-I_{G A B A},
\end{aligned}
$$

where $I_{\text {leak }}$ is a leak current; $I_{N a}$ and $I_{K}$ are voltagegated $\mathrm{Na}^{+}$and $\mathrm{K}^{+}$currents, respectively, which are required for action potential generation; $I_{T}$ is a low threshold T-type $\mathrm{Ca}^{2+}$ current; $I_{C a}$ is a high-threshold $\mathrm{Ca}^{2+}$ current; and $I_{A H P}$ is a $\mathrm{Ca}^{2+}$-activated $\mathrm{K}^{+}$current. $I_{\text {DIC }}$ is a depolarization-activated inward current (DIC), which is activated by the intracellular $\mathrm{Ca}^{2+}$ entering through NMDA receptors (NMDARs). ${ }^{9} I_{N M D A}$ and $I_{G A B A}$ are the NMDA and GABA currents, respectively. The active currents other than $I_{N M D A}$ and $I_{\text {GABA }}$ were taken from previous models of an STN neuron. ${ }^{9,10}$ The NMDA current is described as

$$
I_{N M D A}=g_{N} m_{N} h_{N}\left(V-V_{N M D A}\right),
$$

where $g_{N}$ is the peak conductance, $V_{N M D A}$ is the associated reversal potential, and $m_{N}$ and $h_{N}$ are the activation and inactivation variables, respectively. The variable $m_{N}$ is governed by a pair of first-order equations as follows. ${ }^{11}$

$$
\begin{aligned}
& \frac{d m_{N}}{d t}=\alpha_{m}^{N} x_{N}\left(1-m_{N}\right)-\frac{m_{N}}{\tau_{m}^{N}}, \\
& \frac{d x_{N}}{d t}=\alpha_{x}^{N}\left(1-x_{N}\right) \sum_{j} \delta\left(t-t_{j}^{e x c}\right)-\frac{x_{N}}{\tau_{x}^{N}} .
\end{aligned}
$$

Here, $t_{j}^{e x c}$ denotes the arrival time of the $j$ th cortical input (see below). $\tau_{m}^{N}$ and $\tau_{x}^{N}$ are the time constants to regulate the decay and rise time constants of NMDA activation. The GABA current follows

$$
I_{G A B A}=g_{G}\left(V-V_{G A B A}\right) \text {, }
$$

where $g_{G}$ is the conductance and $V_{G}$ is the reversal potential. The parameters used in the model are selected to reproduce various firing characteristic of STN 
neurons and the voltage-dependent properties of active currents. ${ }^{9,10}$

\subsection{Slow cortical oscillation}

Experimental findings show that during sleep and deep anesthesia, cortical neurons exhibit low-frequency oscillations ( $\square 1 \mathrm{~Hz}$ ) with each cycle composed of two phases. ${ }^{6-8}$ In one phase, which we refer to as the active phase, the depolarization of membrane potential induces high-frequency spiking. In the subsequent phase, which we refer to as the inactive phase, hyperpolarization rapidly suppresses action potential generation. To model the abrupt change between the two phases, we divided time into the intervals corresponding to the active and inactive phases, occurring alternately. We define $T_{A}$ and $T_{I}$ to be the time intervals for the active and inactive phases, respectively. Then, the values of $T_{A}$ and $T_{I}$ are determined as follows:

$$
\begin{aligned}
& T_{A}=\frac{x_{A}}{f_{\mathrm{c}}}\left(1+0.2 y_{A}\right), \\
& T_{I}=\frac{\left(1-x_{A}\right)}{f_{\mathrm{C}}}\left(1+0.2 y_{I}\right),
\end{aligned}
$$

where $X_{A}=0.4$ is the fraction of each cortical period corresponding to the active phase, and $f_{c}=0.7 \mathrm{~Hz}$ is the mean frequency of the slow cortical oscillations. ${ }^{5}$ $y_{A}$ and $y_{I}$ are random variables obtained independently for each cycle from a standard Gaussian distribution.

We also assumed that NMDA activation times $\left(t_{j}^{e x c}\right.$ in Eq. 4) are determined by an inhomogeneous Poisson point process with phase-dependent (firing) rates. The frequency $r$ of the NMDA activation is described as

$$
r= \begin{cases}r_{A}\left(1+0.2 y_{r, 1}\right), & \text { (active phase) } \\ r_{I}\left(1+0.2 y_{r, 2}\right), & \text { (inactive phase) }\end{cases}
$$

where $y_{r, 1}$ and $y_{r, 2}$ are random variables taken from Gaussian distribution. $r_{A}=30 \mathrm{~Hz}$ and $r_{I}=3 \mathrm{~Hz}$ are the mean activation frequencies for the active and inactive phases, respectively.

\section{Results}

We examined the firing dynamics of the STN neuron model driven by low-frequency cortical oscillatory activity. As shown in Fig. 1, burst firing occurred in the presence of GABAergic inhibition, which can produce slow membrane oscillations underlying bursting. ${ }^{1,9,10}$ Higher STN firing rates were found around the times when the active phases of the cortical oscillations occurred (Fig. 1). This result agrees well with the observation that STN bursts occur synchronously with slow cortical oscillations. ${ }^{4,5}$

The increased activation frequency of cortical inputs evoked NMDA activation (Figs. 1A and 1B). While the overall high levels of NMDA activation presumably helped support burst firing, ${ }^{9}$ STN burst onset occurred near the trough of NMDA activation, which built up throughout the period of cortical and STN bursting (Fig. 1C). That is, since NMDA current evolves slowly, STN activity during bursts in the model did not depend on the precise timing of cortical firing.

We also investigated the effects of changing the conductances of GABA and NMDA currents (Fig. 2). Enhancing GABA conductance considerably increased the coefficient of variation (CV) of interspike intervals
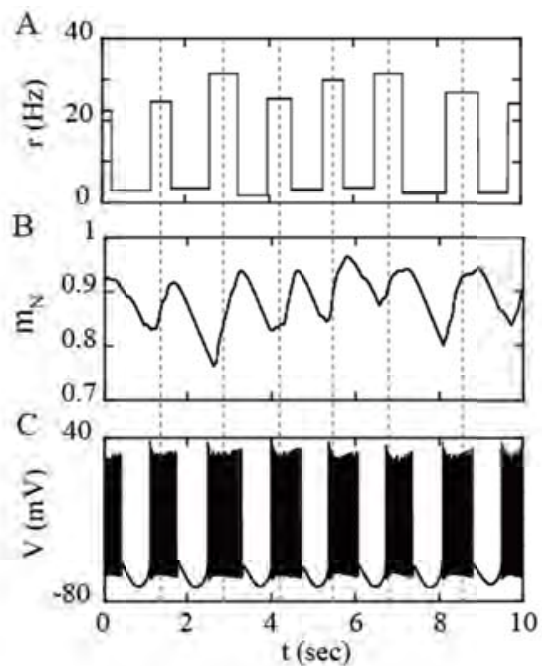

Fig. 1 An example of the response of the STN neuron model for $g_{N}=30$ and $g_{G}=2 \mathrm{mS} / \mathrm{cm}^{2}$. The time courses of the firing rate $r$ of cortical inputs (A), the activation variable $m_{N}$ of the NMDA current (B), and the membrane voltage $V(\mathrm{C})$. The dashed lines show the center of the time period of the active phase of cortical oscillations. 

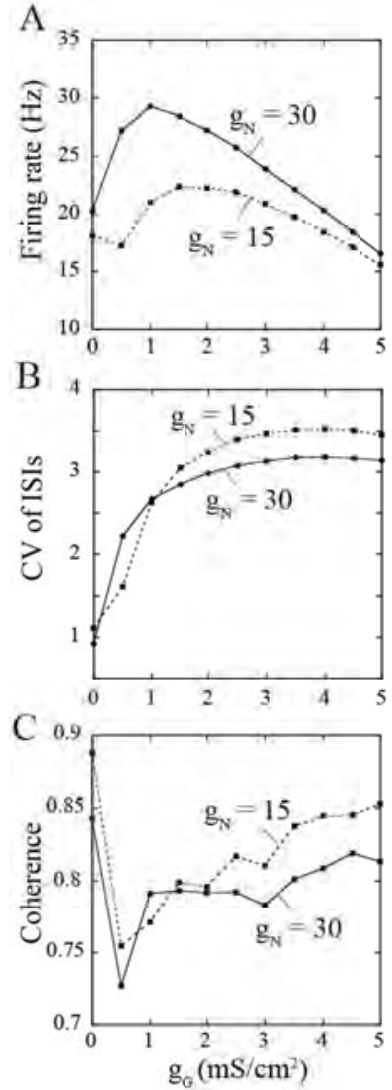

Fig. 2 Changes in the firing rate (A), the coefficient of variation (CV) of interspike intervals (ISIs) (B), and the coherence between the STN and cortical activities at 0.7 $\mathrm{Hz}(\mathrm{C})$ as function of GABA conductance $g_{G}$. The NMDA peak conductance was $g_{N}=15$ (dotted) or 30 (solid) $\mathrm{mS} / \mathrm{cm}^{2}$.

(ISIs) (Fig. 2B), suggesting that GABAergic inhibition plays a key role in the strengthening of burstiness in our model. On the other hand, the increase in NMDA conductances was found to mainly augment the firing rate (Fig. 2A). Interestingly, the induction of burstiness by turning on GABAergic inhibition also tended to decrease the coherence between the cortical inputs and STN firing (Fig. 2C). This result indicates that the enhanced burst firing may prevent the STN neuron from abruptly tracking the change in the cortical activity phases. The depletion of dopamine, a key feature of Parkinson's disease, is known to enhance GABAmediated outward currents in $\mathrm{STN}^{12}$. Based on our results, in the case of dopamine depletion, higher levels of GABA inhibition could suppress the ability of STN to track cortical activity, which may be harmful for coordinating activities between the cortex and basal ganglia.

\section{Conclusions}

In this study, we constructed a model of an STN neuron influenced by cortical slow-wave activity, which reproduced bursting oscillations. The bursting in the model was found to depend not only on NMDA associated with cortical inputs but also on GABAergic inhibition. Because NMDA is a slow current, STN firing times during bursts were not strongly linked to cortical firing times. Indeed, we observed that STN firing during bursts was less coherent than during regular firing (in the absence of GABAergic inputs) and became more irregular with increases in GABA conductance (Fig 2B). These results may suggest a useful framework for how the complex interactions among the STN, cortex and GP shape network activity patterns, particularly in conditions of dopamine depletion when oscillations and bursting are enhanced.

\section{References}

1. M. D. Bevan, P. J. Magill, D. Terman, J. P. Bolam and C. J. Wilson , Move to the rhythm: oscillations in the subthalamic nucleus-external globus pallidus network, Trends Neurosci., 25 (2002) 525-531

2. P. Brown, A. Oliviero, P. Mazzone, A. Insola, P. Tonali and V. Di Lazzaro, Dopamine dependency of oscillations between subthalamic nucleus and pallidum in Parkinson's disease, J. Neurosci., 21 (2001) 1033-1038

3. R. Levy, A. E. Lang, J. O. Dostrovsky, P. Pahapill, J. Romas, J. Saint-Cyr, W. D. Hutchison and A. M. Lozano, Lidocaine and muscimol injections in subthalamic nucleus reverse parkinsonian symptoms, Brain 124 (2001) 2105-2118

4. P. J. Magill, J. P. Bolam and M. D. Bevan, Relationship of activity in the subthalamic nucleusglobus pallidus network to cortical electroencephalogram, J. Neurosci., 20 (2000) 820833

5. P. J. Magill, J. P. Bolam and M. D. Bevan, Dopamine regulates the impact of the cerebral cortex on the subthalamic nucleus-globus pallidus network, Neuroscience, 106 (2001) 313-330 
6. F. Amazica and M. Steriade, Short- and long-range neuronal synchronization of the slow $(<1 \mathrm{~Hz})$ cortical oscillation, J. Neurophysiol., 73 (1995) 2038

7. M. Steriade, F. Amzica and D. Contreras Synchronization of fast $(30-40 \mathrm{~Hz})$ spontaneous cortical rhythms during brain activation, $J$. Neurosci., 16 (1996) 392-417

8. M. Steriade, D. Contreras, F. Amzica and I. Timofeev, Synchronization of fast $(30-40 \mathrm{~Hz})$ spontaneous oscillations in intrathalamic and thalamocortical networks, J. Neurosci., 16 (1996) 2788-2808

9. S. Kubota and J. E. Rubin, NMDA-induced burst firing in a model subthalamic nucleus neuron, $J$. Neurophysiol., 106 (2011) 527-537

10. D. Terman, J. E. Rubin, A. C. Yew and C. J. Wilson, Activity patterns in a model for the subthalamopallidal network of the basal ganglia, $J$. Neurosci., 22 (2002) 2963-2976

11. X. J. Wang, Synaptic basis of cortical persistent activity: the importance of NMDA receptors to working memory, J. Neurosci., 19 (1999) 9587-9603

12. K. Z. Shen and S. W. Johnson, Dopamine depletion alters responses to glutamate and GABA in the rat subthalamic nucleus, Neuroreport, 16 (2005) 171174 